Notfall Rettungsmed 2010 · 13:185-186

DOI 10.1007/s10049-010-1323-x

Online publiziert: 25. April 2010

(c) Springer-Verlag 2010

\author{
H.-R. Arntz ${ }^{1}$ - U. Kreimeier ${ }^{2}$ \\ ${ }^{1}$ Medizinische Klinik II; Kardiologie und Pulmonologie, \\ Charité Campus Benjamin Franklin, Berlin \\ ${ }^{2}$ Klinik für Anaesthesiologie, Klinikum der Universität München
}

\title{
Die neuen Leitlinien zur kardiopulmonalen Reanimation und Notfall- behandlung des akuten Koronarsyndrom
} Was werden sie bringen?

Mit zunehmender Spannung wird der Consensus of Science 2010 des International Liaison Committee on Resuscitation (ILCOR) und die daraus entwickelten für uns verbindlichen Leitlinien des European Resuscitation Council (ERC) erwartet. Am 18. Oktober wird es soweit sein. Ende des Jahres 2010 wird auch die deutsche Übersetzung voraussichtlich in „Notfall + Rettungsmedizin“ verfügbar sein.

Vier Arbeiten in diesem Heft geben wichtige Grundinformationen, die in der Entwicklung und Diskussion im ILCORProzess und in den neuen Leitlinien eine wesentliche Rolle spielen. Die Arbeiten werfen auch ein Schlaglicht auf die Komplexität des Themas. Zum Beispiel spricht die Anzahl unterschiedlicher Defibrillatoren mit verschiedensten Arbeitskonzepten, Energielevel, differenzierten Entladungscharakteristika und anderen Modifikationen Bände über das Problem, eindeutige oder zumindest sehr verlässliche Angaben über die optimale Defibrillationsstrategie zu entwickeln. Eine ähnliche Problematik finden wir bei Betrachtung mechanischer Reanimationssysteme, von denen jedes einzelne hinsichtlich hämodynamischer Daten im Experiment vielversprechend ist.
Der klinische Effizienznachweis im Sinne einer höheren Überlebensrate bei kardiopulmonaler Reanimation in kontrollierten randomisierten Untersuchungen - die Studien zu verblinden, ist schwierig oder unmöglich - erweist sich in Anbetracht der unübersehbaren Vielfalt nicht kontrollierbarer Parameter, die das Reanimationsergebnis beeinflussen können, als extrem schwierig.

\section{(7) Mindestbedingung für eine Durchführung von klinischen Studien mit neuen Geräten ist eine hohe Fallzahl}

Die Mindestbedingung für die Durchführung von ersten klinischen Studien mit neuen Geräten im Vergleich zur konventionellen Reanimation ist daher eine hohe Fallzahl. Sollten die Ergebnisse mit unterschiedlichen Geräten, z. B. Defibrillatoren oder mechanischen Reanimationssystemen jeweils erfolgreich sein, wäre in einem nächsten Schritt der sog. „head to head“-Vergleich zwischen den Konkurrenten $\mathrm{zu}$ fordern. Ist schon die Durchführung des ersten Schritts nur in sehr seltenen Fällen wegen der enormen Kosten für eine Herstellerfirma oder einen Sponsor zu stemmen, ist der zweite Schritt selbst bei maximalem Engagement der Rettungsdienste als praktisch illusionär zu bezeichnen. Hinzu kommen ethische und formale Hinderungsgründe bei der Durchführung von Studien zur kardiopulmonalen Reanimation. Die Unterschiede in den Rettungssystemen, insbesondere bei der aus Fallzahlgründen notwendigen globalen Betrachtung, sind erheblich und verhindern auf mancherlei Gebiet eine weltweite Kooperation. Ethische Bedenken bei Studien an nicht einwilligungsfähigen Patienten sind ein sehr ernster Aspekt, der eigentlich eine sehr intensive Auseinandersetzung der Problematik erfordern würde. Leider ist bei Ethikkommissionen eine zunehmende Tendenz zu beobachten, Studien im Zusammenhang mit der kardiopulmonalen Reanimation ohne Betrachtung der inhaltlichen Problematik und ohne nähere Begründung rundweg abzulehnen. Dies gilt auch für Untersuchungen z. B. an Patienten, die aufgrund ihrer Schmerzsituation oder notwendigen Behandlung mit starken Schmerzmitteln bei akutem Koronarsyndrom oder nach schwerer Verletzung nur sehr begrenzt einwilligungsfähig sind. 
Es ist leicht nachvollziehbar, dass der resultierende Mangel an verlässlichen Daten, zumal aus der frühen präklinischen Behandlungsphase, die Erstellung von Leitlinien und Empfehlungen für die Erstversorgung erheblich erschwert, und in vielen Fällen die Notwendigkeit von Analogieschlüssen oder Extrapolierung von Ergebnissen aus unter anderen Bedingungen durchgeführten Studien notwendig macht. Dies bedeutet für die Arbeitsgruppen im ILCOR-Prozess eine große Verantwortung, denn die praktisch Handelnden im Rettungsdienst und auch in der Notaufnahme benötigen diese Hinweise. Dennoch ist zu hoffen und zu erwarten, dass wir auch 2010 wieder Leitlinien an die Hand bekommen werden, die denjenigen, die die unmittelbare Verantwortung für die Versorgung der Notfallpatienten vor Ort tragen, die notwendige Sicherheit geben, nach neuesten und gesicherten Erkenntnissen und damit richtig im Sinne für die Erhaltung des Lebens und der Gesundheit ihrer Patienten zu handeln.

Ihre

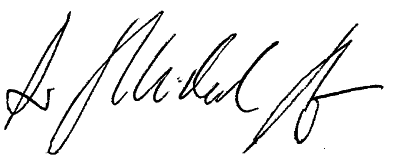

H.-R. Arntz

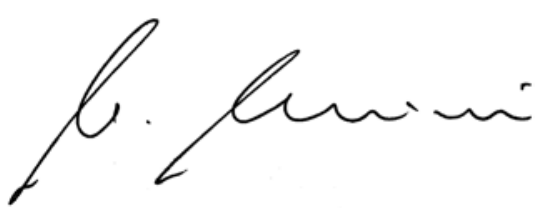

U. Kreimeier

\section{Korrespondenzadresse}

\section{Prof. Dr. H.-R. Arntz}

Medizinische Klinik Il; Kardiologie und Pulmonologie, Charité Campus Benjamin Franklin Hindenburgdamm 30, 12200 Berlin

hans-richard.arntz@charite.de
„Notfall + Rettungsmedizin“ bietet Ihnen 8-mal im Jahr umfassende und aktuelle Beiträge zu interessanten Themenschwerpunkten (Leitthemen) aus allen Bereichen der Notfallmedizin.

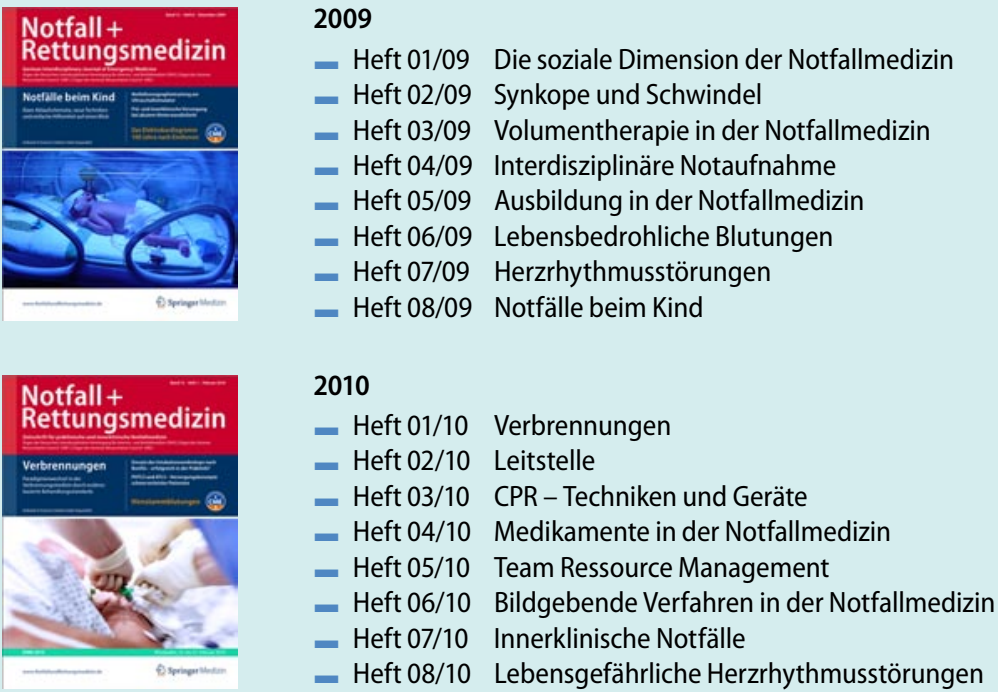

Notfall +

Rettungsmedizin

Leitstelle

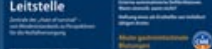

mind

Heft 08/10 Lebensgefährliche Herzrhythmusstörungen

Alle Beiträge auf einen Blick

Abonnenten haben online Zugriff auf alle Beiträge im elektronischen Volltextarchiv unter www.NotfallundRettungsmedizin.de

Ins Volltextarchiv gelangen Sie von der Startseite über den Navigationspunkt "Online-Archiv".

Eine interessante Lektüre wünscht Ihnen

Ihre Redaktion "Notfall + Rettungsmedizin" 